\title{
CALIDAD DE SEMILLA DE GENOTIPOS ISOCITOPLASMICOS DE SORGO (Sorghum bicolor L. Moench.) ${ }^{1}$
}

\author{
Víctor Pecina ${ }^{2}$,Enrique Navarro ${ }^{2}$, Héctor Williams ${ }^{2}$, Raúl Rodríguez ${ }^{2}$
}

\begin{abstract}
RESUMEN
Calidad de semilla de genotipos isocitoplasmicos de sorgo (Sorghum bicolor L. Moench.). La producción de híbridos de sorgo depende en la actualidad del uso de un solo sistema de esterilidad génico-citoplásmica (Milo-Kafir, Citoplasma A1). Por diversificar el número de fuentes, se han encontrado nuevos tipos como el denominado Citoplasma A2, el cual fue introducido para su estudio al Campo Experimental de Río Bravo, Tamaulipas, México (INIFAP-CIRNE), donde se formaron los diferentes genotipos isocitoplásmicos y se produjo la semilla utilizada en el presente trabajo, el cual tiene como objetivo: determinar si existen diferencias en calidad de semilla que pudieran ser debidas al tipo de citoplasma (A1 y A2). El diseño experimental fue completamente al azar en un arreglo factorial y dos repeticiones. Los resultados indicaron que no existen diferencias entre los dos sistemas de androesterilidad (A1 y A2) en las características de peso volumétrico, peso de mil semillas, germinación (G) y peso seco de plantula (PSP) a través de nueve meses de almacenamiento, mientras que en vigor ( $\mathrm{G}$ y PSP después de envejecimiento acelerado) el citoplasma A2 superó al A1, el mismo comportamiento se observó en la 'semilla cosechada a madurez fisiológica en tanto que a madurez de cosecha el A1 se muestra superior en G, PSP y PSPEA.
\end{abstract}

\begin{abstract}
Seed quality of sorghum (Sorghum bicolor L. Moench) isocytoplasmic genotypes. The production of sorghum hybrids depends almost entirely on one genic-cytoplasmic sterility system (Milo-Kafir, A1 Cytoplasm), therefore its importance in increasing the diversity of the number of male-sterile sources, as the named A2 Cytoplasm. This system was introduced at the Rio Bravo Experiment Station (INIFAP-CIRNE) in Tamaulipas, Mexico, where ge-netic material and seed production were developed. The objetive was to compare the seed quality ofthe isocytoplasmic genotypes (A1 and A2 cytoplasm). A Completely Radomized Experimental Design with Factorial Arrangement and two replications was used. The results show that there were no differences between the two types of cytoplasm (A1 and A2) for the following quality traits: volumetric weight of seeds, 1000 seeds' weight, germination (G) and seedling dry weight (SDW), through nine months of storage; whereas in vigor (G and SDW after fast aging) the A2 cytoplasm surpassed the Al cytoplasm and the same behavior was observed on the seed harvested at its physiological maturity, while at harvest maturity the $\mathrm{Al}$ was superior in $\mathrm{G}$ and SDW.
\end{abstract}

\section{INTRODUCCION}

La falta de variabilidad genética y la uniformidad en los cultivos ha provocado severos problemas en el pasado, en especies como la papa en Irlanda en 1845-46 al ser afectada por la enfermedad Phythophtora infestans, en maíz en 1970 con Helminthosporium maydis y más recientemente en trigo y café con las rayas, por lo que desde 1972 el Consejo Nacional de Investigación de los Estados Unidos de América, dependiente de la Academia Nacional de Ciencias alertó de que el cultivo de sorgo presenta una gran vulnerabilidad debido a que casi la

\footnotetext{
1 Presentado en la XL Reunión Anual del PCCMCA en Costa Rica, América Central. 13 al 19 de marzo, 1994.

2 Estudiante de Maestría en Tecnología de Semillas Actualmente Investigador del SARH-INIFAP-CIRNE. Ebano, S. L. P., México. C.P. 79150 Apdo. Postal 87.

3 Profesor Investigador del Dpto de Fitomejoramiento de la UAAAN en Saltillo, Coahuila; México.

4 Investigadores del Programa de Sorgo de SARH-INIFAP-CIRNE. Río Bravo, Tamaulipas, México. C.P. 88900 Apdo. Postal 172.
} 
totalidad de los híbridos dependen del uso de un solo sistema de androesterilidad, por lo que existe el peligro de que una enfermedad pueda causar estragos en este cultivo. Ante esta situación se han buscado nuevas fuentes de esterilidad masculina distintas a la proveniente de Milo (citoplasma A1); por lo que Ross y Hackerott (1972) liberan siete líneas androestériles con nuevos citoplasmas los denominados KS34 y KS39 provenientes de diferentes especies de sorgo y cuyas características de androesterilidad eran similares al Milo. Posteriormente al evaluar su comportamiento agronómico en los híbridos Ross y Kofoid (1979) señalan que no existen diferencias estadísticas entre ambos en las características de días a floración, altura de planta, rendimiento de grano, panojas por planta, peso de 1000 semillas y número de semillas por panoja. Además no se detectó ningún efecto adverso del citoplasma en el comportamiento agronómico de las cruzas; a conclusiones similares llegan en 1981 Lenz y Atkins, sin embargo dichos citoplasmas no han sido utilizados aún en la producción comercial de híbridos.

En la actualidad se han identificado nuevos sistemas de androesterilidad diferentes a la proveniente del Milo, a los que se les ha designado como citoplasma A2 (IS 12662C); A3 (IS1112C) y A4 (IS7920C) según lo consigna Schertz (1983).

El sistema A2 fue introducido para su estudio al Campo Experimental de Río Bravo, Tamaulipas, México, en 1982, estudiando primero su tipo de reacción en las líneas élite y comerciales más sobresalientes del programa de sorgo; esto permitió formar líneas isocitoplásmicas con los dos sistemas de androesterilidad (A1 y A2), y por consiguiente se formaron también híbridos (Williams, 1987); estos genotipos ya han sido utilizados en estudios para determinar su comportamiento agronómico; Rodríguez et al. (1991) no detectaron diferencias estadísticas entre ambos sistemas para las características de días a floración, altura de planta, longitud de panoja, excersión y rendimiento de grano, por lo cual concluyen que el citoplasma A2 presenta un potencial de uso similar al del A1 (Milo), sin embargo no se ha realizado ningún estudio para determinar si el citoplasma A2 tiene algún efecto en la calidad de la semilla de sorgo, por lo que se planteó la presente investigación con el objetivo de determinar si existen diferencias entre los dos citoplasmas (A1 y A2) que pudieran influir en la calidad de la semilla obtenida.

\section{MATERIALES Y MÉTODOS}

El presente estudio se llevó a cabo en el Laboratorio de Control de Calidad del Centro de Capacitación y Desarrollo de Tecnología de Semillas (CCDTS) de la Universidad Autónoma Agraria Antonio Narro (UAAAN) en Saltillo, Coahuila, México.

El material genético utilizado fue desarrollado en el ciclo otoño-invierno de 1989-90 en el Campo Experimental de Río Bravo, Tamaulipas, México (INIFAP-CIRNE) y corresponde a los siguientes materiales: la línea LRB-104A, y los híbridos LRB-102 x LRB-63, LRB-104 x LRB-63, LRB-104 x LRB-25 y LRB-104 x SCO-599-11E; todos en sus dos versiones de androesterilidad (citoplasma A1 y A2).

Se evaluaron un total de 80 tratamientos que resultan de la combinación de cinco genotipos, con dos citoplasmas (A1 y A2), en dos épocas de cosecha (madurez fisiológica y madurez de cosecha) y cuatro periodos de almacenamiinto (0, 3, 6 y 9 meses).

El diseño experimental fue completamente al azar con arreglo factorial y dos repeticiones.

Para obtener la semilla del presente trabajo se establecieron lotes individuales de cada una de las líneas androesteriles (LRB-102A y LRB-104A), un lote por cada versión (A1 y A2), realizándose polinizaciones manuales con los restauradores LRB-25, LRB-63 y SCO599-11E; al final en la cosecha se juntaron en un solo compuesto cada material según su genealogía. Cabe mencionar que debido a que los genotipos de sorgo presentaron diferente grado de madurez y dada la cantidad de materiales que se estuvieron trabajando se establecieron diferentes fechas de siembra para lograr cubrir el periodo de floración de las hembras, por lo que, la cosecha a madurez fisiológica se llevó a cabo durante un periodo de 15 días y una vez cosechada la semilla de cada genotipo se almacenó en bolsas de papel en un cuarto frío $\left(10^{\circ} \mathrm{C}\right)$, hasta tener todos los materiales bajo la misma condición, lo cual se prolongó por 
aproximadamente 30 días, hasta cosechar el tratamiento de madurez de cosecha. Posteriormente la semilla fue secada hasta alcanzar un $10 \%$ de humedad, después de lo cual se evaluó la calidad inicial. Por otra parte se almacenó una pequeña muestra de semilla de cada genotipo (100 g) bajo condiciones naturales en el Laboratorio de Producción de Semillas del CCDTS de la UAAAN en Saltillo, Coahuila, México, muestreándose la semilla cada tres meses para observar su comportamiento.

\section{RESULTADOS}

Los análisis de varianza detectaron diferencias altamente significativas en todas las variables evaluadas, por lo cual se realizó una partición de los efectos a través de contrastes ortogonales, observándose diferencias muy marcadas entre los genotipos evaluados (Cuadro 1), y al comparar la línea androestéril LRB- 104 con los cuatro híbridos, resultan dos de ellos superiores al resto, mientras que la línea iguala o supera en calidad a los híbridos generados con el restaurador LRB-63. En cuanto al tipo de citoplasma los análisis no detectaron diferencias estadísticas entre los dos sistemas (A1 y A2) en los atributos de calidad para peso volumétrico (PV), peso de 1000 semillas (PMS), germinación estándar (G), y peso seco de plántula (PSP) en diferentes periodos de almacenamiento, sin embargo al someterlos a un envejecimiento acelerado el sistema A2 exhibió un comportamiento superior al A1, conservando una mayor capacidad de germinación (Cuadro 2), en peso seco de plántula no se observó tal efecto.

En cuanto a la interacción genotipo-citoplasma, el análisis de varianza detectó que los genotipos presentan respuestas medias estadísticamente diferentes al combinarse con uno u otro sistema de androesterilidad (citoplasma A1 y A2) en todas las características evaluadas bajos las dos condiciones, con excepción de peso seco de plántula después de envejecimiento acelerado bajo el citoplasma A2 (Cuadros 3 y 4), además pudo observarse en la comparación de medias (Duncan a $0,05)$ que el comportamiento medio de la línea (LRB104A) es igual al mejor de los híbridos bajo el citoplasma

Cuadro 1. Medias de seis características de calidad de semilla de cinco genotipos de sorgo.

\begin{tabular}{lcccccc}
\hline Genotipo & PV & PMS & G & PSP & GEA & PSPEA \\
\hline LRB-104A & $73 \mathrm{~b}$ & $28 \mathrm{a}$ & $85 \mathrm{~b}$ & $8,0 \mathrm{c}$ & $82 \mathrm{~b}$ & $6,8 \mathrm{~b}$ \\
LRB-104 x SCO-599-11E & $77 \mathrm{a}$ & $28 \mathrm{a}$ & $90 \mathrm{a}$ & $8,7 \mathrm{~b}$ & $86 \mathrm{a}$ & $7,0 \mathrm{~b}$ \\
LRB-104 x LRB-25 & $73 \mathrm{~b}$ & $27 \mathrm{a}$ & $91 \mathrm{a}$ & $9,1 \mathrm{a}$ & $80 \mathrm{~b}$ & $7,7 \mathrm{a}$ \\
LRB-104 x LRB-63 & $72 \mathrm{~b}$ & $29 \mathrm{a}$ & $87 \mathrm{~b}$ & $8,8 \mathrm{~b}$ & $73 \mathrm{c}$ & $7,1 \mathrm{~b}$ \\
LRB-102 x LRB-63 & $71 \mathrm{~b}$ & $24 \mathrm{~b}$ & $87 \mathrm{~b}$ & $7,8 \mathrm{c}$ & $67 \mathrm{~d}$ & $6,5 \mathrm{c}$ \\
\hline
\end{tabular}

Valores en la misma columna con distinta letra son diferentes estadísticamente al 0,05\% de probabilidad (Duncan).

Cuadro 2. Medias de seis características de calidad de semilla de acuerdo con el sistema de androesterilidad en sorgo.

\begin{tabular}{lcccccc}
\hline Fuente & $\mathbf{P V}(\mathbf{k g} / \mathbf{h l})$ & $\mathbf{P M S}(\mathbf{g})$ & $\mathbf{G}(\%)$ & $\mathbf{P S P}(\mathbf{m g})$ & $\mathbf{G}(\%)$ & PSPEA(mg) \\
\hline Citoplasma A1 & $73 \mathrm{a}$ & $27 \mathrm{a}$ & $88 \mathrm{a}$ & $8,6 \mathrm{a}$ & $74 \mathrm{~b}$ & $6,8 \mathrm{a}$ \\
Citoplasma A2 & $73 \mathrm{a}$ & $27 \mathrm{a}$ & $89 \mathrm{a}$ & $8,4 \mathrm{a}$ & $82 \mathrm{a}$ & $7,2 \mathrm{a}$ \\
\hline
\end{tabular}

Valores en la misma columna con distinta letra son diferentes estadísticamente al 0,05\% de probabilidad (Duncan). 
Cuadro 3. Comportamiento medio de cinco genotipos de sorgo en calidad de semilla bajo el citoplasma A1.

\begin{tabular}{lcccccc}
\hline Genotipo & PV & PMS & G & PSP & GEA & PSPEA \\
\hline LRB-104A & $72 \mathrm{~b}$ & $27 \mathrm{~b}$ & $78 \mathrm{c}$ & $6,8 \mathrm{~b}$ & $78 \mathrm{~b}$ & $6,4 \mathrm{~b}$ \\
LRB-104 x SCO-599-11E & $77 \mathrm{a}$ & $29 \mathrm{a}$ & $91 \mathrm{a}$ & $8,3 \mathrm{a}$ & $91 \mathrm{a}$ & $6,9 \mathrm{~b}$ \\
LRB-104 x LRB-25 & $71 \mathrm{~b}$ & $27 \mathrm{~b}$ & $93 \mathrm{a}$ & $9,3 \mathrm{a}$ & $70 \mathrm{c}$ & $8,0 \mathrm{a}$ \\
LRB-104 x LRB-63 & $73 \mathrm{~b}$ & $29 \mathrm{a}$ & $91 \mathrm{a}$ & $9,6 \mathrm{a}$ & $73 \mathrm{~b}$ & $7,4 \mathrm{a}$ \\
LRB-102 x LRB-63 & $70 \mathrm{~b}$ & $24 \mathrm{c}$ & $86 \mathrm{~b}$ & $7,8 \mathrm{~b}$ & $53 \mathrm{~d}$ & $5,3 \mathrm{c}$ \\
\hline
\end{tabular}

Valores en la misma columna con distinta letra son diferentes estadísticamente al 0,05\% de probabilidad (Duncan).

Cuadro 4. Comportamiento medio de cinco genotipos de sorgo en calidad de semilla bajo el citoplasma A2.

\begin{tabular}{lcccccc}
\hline Genotipo & PV & PMS & G & PSP & GEA & PSPEA \\
\hline LRB-104A & $74 \mathrm{a}$ & $28 \mathrm{a}$ & $90 \mathrm{a}$ & $9,2 \mathrm{a}$ & $85 \mathrm{a}$ & $7,2 \mathrm{a}$ \\
LRB-104 x SCO-599-11E & $76 \mathrm{a}$ & $27 \mathrm{a}$ & $89 \mathrm{a}$ & $8,1 \mathrm{~b}$ & $81 \mathrm{~b}$ & $7,9 \mathrm{a}$ \\
LRB-104 x LRB-25 & $74 \mathrm{a}$ & $27 \mathrm{a}$ & $91 \mathrm{a}$ & $9,0 \mathrm{a}$ & $88 \mathrm{a}$ & $7,3 \mathrm{a}$ \\
LRB-104 x LRB-63 & $70 \mathrm{~b}$ & $29 \mathrm{a}$ & $83 \mathrm{~b}$ & $8,1 \mathrm{~b}$ & $73 \mathrm{c}$ & $6,8 \mathrm{a}$ \\
LRB-102 x LRB-63 & $71 \mathrm{~b}$ & $24 \mathrm{~b}$ & $88 \mathrm{a}$ & $7,8 \mathrm{~b}$ & $80 \mathrm{~b}$ & $7,6 \mathrm{a}$ \\
\hline
\end{tabular}

Valores en la misma columna con distinta letra son diferentes estadísticamente al 0,05\% de probabilidad (Duncan).

A2 y se muestra también que el genotipo LRB-102 x LRB-63 no varía en su respuesta bajo las dos condiciones aún y cuando presente los menores valores de calidad de semilla, el resto de los híbridos modifica su comportamiento al combinarse con los citoplasmas A1 y A2.

Con respecto a la época de cosecha se observó diferencias altamente significativas en todas las características de calidad evaluadas, presentando una mayor calidad de semilla el sistema de androesterilidad A2, cuando ésta es cosechada a madurez fisiológica, mientras que a madurez de cosecha sucede una tendencia inversa (Cuadros 5 y 6) siendo superior el citoplasma A1.

En lo que se refiere a el efecto del almacenamiento sobre la calidad de la semilla, se pudo detectar diferencias altamente significativas entre los periodos de muestreo en el porcentaje de germinación y peso seco de plántula, detectando la comparación de medias (Cuadro 7) que la máxima capacidad de germinación y peso seco de plántula se alcanzan a los tres meses después de evaluada la calidad inicial, posteriormente hay una disminución gradual de ésta de acuerdo con el tiempo de almacenamiento; el mismo comportamiento es exhibido por los diferentes genotipos. En cuanto a la interacción periodo de almacenamiento-citoplasma no se detectaron diferencias estadísticas entre los dos sistemas de esterilidad génico-citoplásmica (A1 y A2), como se puede observar en el Cuadro 8.

En cuanto a los coeficientes de variación, se puede observar que son bajos, pues el más alto lo presenta la variable peso seco de plántula después de envejecimiento acelerado con $10,19 \%$.

\section{DISCUSION}

Las diferencias en peso volumétrico, peso de mil semillas, así como en porcentaje de germinación y peso 
Cuadro 5. Comportamiento medio de dos sistemas de androesterilidad en calidad de semilla de sorgo cosechada a madurez fisiológica.

\begin{tabular}{lcccccc}
\hline Fuente & PV & PMS & G & PSP & GEA & PSPEA \\
\hline Citoplasma A1 & $73 \mathrm{~b}$ & $28 \mathrm{a}$ & $80 \mathrm{~b}$ & $7,5 \mathrm{~b}$ & $74 \mathrm{~b}$ & $6,7 \mathrm{~b}$ \\
Citoplasma A2 & $75 \mathrm{a}$ & $26 \mathrm{a}$ & $88 \mathrm{a}$ & $8,2 \mathrm{a}$ & $87 \mathrm{a}$ & $8,7 \mathrm{~b}$ \\
\hline
\end{tabular}

Valores en la misma columna con distinta letra son diferentes estadísticamente al 0,05\% de probabilidad (Duncan).

Cuadro 6. Comportamiento medio de dos sistemas de androesterilidad en calidad de semilla de sorgo cosechada a madurez de cosecha.

\begin{tabular}{lcccccc}
\hline Fuente & PV & PMS & G & PSP & GEA & PSPEA \\
\hline Citoplasma A1 & $73 \mathrm{a}$ & $27 \mathrm{a}$ & $94 \mathrm{a}$ & $9,7 \mathrm{a}$ & $76 \mathrm{a}$ & $6,9 \mathrm{a}$ \\
Citoplasma A2 & $71 \mathrm{a}$ & $26 \mathrm{a}$ & $89 \mathrm{~b}$ & $8,7 \mathrm{~b}$ & $76 \mathrm{a}$ & $5,7 \mathrm{~b}$ \\
\hline
\end{tabular}

Valores en la misma columna con distinta letra son diferentes estadísticamente al 0,05\% de probabilidad (Duncan).

Cuadro 8. Medias de germinación y peso seco de plántula de acuerdo con el periodo de almacenamiento, promedio de cinco genotipos isocitoplásmicos de sorgo.

\begin{tabular}{ccc}
\hline Almacenamiento(meses) & Germinación(\%) & Peso seco de plantula(mg) \\
\hline 0 & $88 \mathrm{~b}$ & $9,0 \mathrm{a}$ \\
3 & $92 \mathrm{a}$ & $9,5 \mathrm{a}$ \\
6 & $87 \mathrm{~b}$ & $8,2 \mathrm{~b}$ \\
9 & $84 \mathrm{~b}$ & $7,4 \mathrm{c}$ \\
\hline
\end{tabular}

Valores en la misma columna con distinta letra son diferentes estadísticamente al 0,05\% de probabilidad (Duncan).

Cuadro 9. Comportamiento de la calidad de semilla de dos sistemas de androesterilidad en sorgo, a través de cuatro periodos de almacenamiento.

\begin{tabular}{|c|c|c|c|c|c|c|c|c|}
\hline \multirow[t]{2}{*}{ Fuente } & \multicolumn{2}{|c|}{0 meses } & \multicolumn{2}{|c|}{3 meses } & \multicolumn{2}{|c|}{6 meses } & \multicolumn{2}{|c|}{9 meses } \\
\hline & $\mathbf{G}$ & PSP & $\mathbf{G}$ & PSP & $\mathbf{G}$ & PSP & $\mathbf{G}$ & PSP \\
\hline CitoplasmaA1 & $88 \mathrm{a}$ & $9,0 \mathrm{a}$ & $92 \mathrm{a}$ & $9,6 \mathrm{a}$ & $88 \mathrm{a}$ & $8,2 \mathrm{a}$ & $84 \mathrm{a}$ & $7,5 \mathrm{a}$ \\
\hline CitoplasmaA2 & 89 a & $9,0 \mathrm{a}$ & 94 a & $9,4 \mathrm{a}$ & $88 \mathrm{a}$ & $8,1 \mathrm{a}$ & $86 \mathrm{a}$ & $7,2 \mathrm{a}$ \\
\hline
\end{tabular}

Valores en la misma columna con distinta letra son diferentes estadísticamente al 0,05\% de probabilidad (Duncan). 
seco de plántula a diferentes periodos de almacenamiento y después de envejecimiento acelerado (Cuadro 1) parece ser lo normal entre genotipos, ya que dichos materiales difieren en su composición genética, además se está evaluando y comparando una línea endocriada (LRB104A), con cuatro híbridos, por lo que debido a la heterosis algunos de ellos presentaron valores más elevados en las características de calidad que se evaluaron; asi mismo se observó que tal línea presenta buenos atributos de calidad, pues iguala o supera en algunas características (Cuadro 1) a los dos peores híbridos, lo cual es benefico ya que puede buscarse la combinación apropiada con un restaurador de tal manera que se puedan formar híbridos superiores en calidad de semilla cuando esto sea necesario.

En cuanto a los sistemas de androesterilidad los análisis no detectaron diferencias estadísticas entre el citoplasma A1 y A2 (Cuadro 2) en las características de calidad para peso volumétrico, peso de mil semillas, en germinación estándar y peso seco de plántula antes y después de envejecimiento acelerado; en germinación después de envejecimiento acelerado sí se detectó significancias, sin embargo todo lo anterior ratifica lo encontrado por Rodríguez et al. (1991) al estudiar estos dos sistemas de androesterilidad, en cuanto a características agronómicas utilizando estos genotiposy confirma también que es posible utilizar el citoplasma A2 en la producción de híbridas pues su comportamiento es similar al del A1 en cuanto a calidad de semillas.

Con respecto a la interacción genotipo-citoplasma (Cuadro 3 y 4) se pudo observar que existe una tendencia definida en la respuesta de cada material hacia un sistema u otro, por lo que según su comportamiento en calidad de semilla (mayor o menor), ésta puede producirse en la versión que ofrezca la mayor calidad, lo que permitirá comercializar semilla de la más alta calidad y a la vez disminuir los problemas durante su almacenamiento y conservación.

En lo que se refiere a la época de cosecha, se detectaron grandes diferencias entre éstas en las características de peso volumétrico, peso de mil semillas y en germinación y peso seco de plántula después de envejecimiento acelerado, presentando una mayor calidad la semilla cosechada a madurez fisiológica, lo cual se ajusta a lo esperado según los reportes revisados (Deluoche, 1964), en donde se menciona que la semilla cosechada a madurez fisiológica poseé el máximo número de atributos de calidad, mientras que a madurez de cosecha la semilla puede presentar un cierto grado de deterioro en su calidad, debido a que durante este periodo que transcurre de una etapa a otra, pueden presentarse condiciones adversas de clima que pueden afectar directamente la calidad. Por otra parte al evaluar la semilla cosechada durante estas dos épocas a diferentes periodos de almacenamiento el comportamiento de calidad no es el mismo, por lo que se atribuye tal respuesta al efecto que pudieron tener los altos contenidos de humedad en la semilla cosechada a madurez fisiológica, ya que éstas permanecieron en un cuarto frío después de ser cosechadas por un espacio de tiempo considerable hasta ser secadas, por lo tanto las condiciones de almacenamiento no fueron las adecuadas aún y cuando la temperatura si fue la óptima $\left(10^{\circ} \mathrm{C}\right)$.

En cuanto a la interacción citoplasma-época de cosecha se observó que el sistema de androesterilidad A2 supera en calidad al citoplasma A1 (Cuadro 5 y 6) cuando la semilla es cosechada a madurez fisiológica, mientras que a madurez de cosecha la tendencia es inversa siendo superior el A1, tales resultados pueden atribuirse a que posiblemente las condiciones ambientales que prevalecieron en el campo después de madurez fisiológica afectaron más a las semillas con citoplasma A2.

Por otra parte en lo que se refiere al efecto del almacenamiento sobre la calidad de semilla se observó que la máxima capacidad germinativa y peso seco de plántula se alcanzó a los tres meses después de evaluada la calidad inicial (Cuadro 7), posteriormente existe una disminución gradual de acuerdo con el tiempo de almacenamiento, se presume que tal comportamiento pudo deberse a una dormancia de tipo secundario que pudo ser impuesta cuando la semilla fue sometida a un periodo de bajas temperaturas $\left(10^{\circ} \mathrm{C}\right.$ por 30 días $)$ después 
de estar soportando temperaturas superiores a los $30^{\circ} \mathrm{C}$ y a un error en el muestreo pues el tamaño de muestra no fue el adecuado.

En cuanto a los coeficientes de variación se pudo observar que son bajos, pués el más alto es del $10 \%$, por lo tanto se puede considerar que la información es bastante confiable.

\section{CONCLUSIONES}

Con base a los resultados obtenidos y bajo las condiciones en que se desarrolló el trabajo, se concluye que:

1. No se detectaron diferencias entre los dos sistemas de androesterilidad (citoplasma A1 y A2) en los atributos de calidad para peso volumétrico, peso de mil semillas, germinación y peso seco de plántula. En vigor (GEA y PSPEA) el citoplasma A2 exhibió un mejor comportamiento que el A1.

2. En cuanto a la época de cosecha se observó que a madurez fisiológica, la semilla con citoplasma A2 presenta una mayor calidad que el A1, mientras que a madurez de cosecha sucede una tendencia inversa en tres de las características (G, PSP y PSPEA).

3. Los resultados muestran que la utilización del sistema de androesterilidad génico-citoplásmica A2 presenta un potencial de uso similar al del sistema A1.

4. Para confirmar lo anterior és necesario continuar con este tipo de estudios, procurando disponer de una mayor cantidad de semilla para evitar posibles errores en el muestreo.

\section{LITERATURA CITADA}

ANONYMOUS. 1972. Genetic vulnerability of major crops. Washington D.C. U.S.A. Nat. Acad. of Sci. p 156-71.

DELOUCHE, J. C. 1964. Seed Maturation. Seed Technology Laboratory Mississippi State University, prepared for international training. Mississippi. U.SA

LENZ, M. c.; R. E. ATKINS. 1981. Comparisions of agronomic and morphologic characters in sorghums having different cytoplasm. Crop. Sci. 21 :946-950.

RODRIGUEZ, H.R.; H. WILLlAMS, A.; H. TORRES, M. 1991. Comparación agronómica de dos tipos de esterilidad génico-citoplásmica en sorgo. Campo Experimental Río Bravo. SARH.INIFAP. In: Memorias del II Congreso Nacional de Génetica. Saltillo, Coahuila, México. p 19.

ROSS, W.M.; H.L. HACKEROIT. 1972. Registration of seven isocytoplasmic sorghum germoplasm lines. Crop. Sci. 12:720.

; K.D. KOFOID. 1979. Effect of non-milo cytoplasm on the agronomic performance ofsorghum. Crop. Sci. 19:167-170.

SCHERTZ, K.F. 1983. Cytoplasms and potential for reduction of genetic vulnerability in sorghum. In: Proccedingsof the plant breeding methods and Approches in sorghum workshop for Latin America. Sponsored by INTSORMIL-INIA-ICRISA T. p 114-120,

WILLIAMS, H.A. 1987. Respuesta de líneas experimentales y comerciales de sorgo al sistema de androesterilidad génico-citoplásmica A2. Fitotecnia. México. 10:91-98. 